\title{
Linking virulence and transmission in malaria
}

To ensure transmission to the next human host, the malaria parasite Plasmodium falciparum must differentiate from asexually replicating parasites, which proliferate in human erythrocytes, to sexual gametocytes, which are taken up by mosquitoes during a blood meal. Furthermore, asexual parasites evade host immune responses by regularly switching the expression of antigens on the surface of infected erythrocytes. Although epigenetic modifications are suggested to be involved in regulating both sexual differentiation and antigenic variation, the mechanistic basis of this is not fully understood. Two studies now identify the P. falciparum proteins heterochromatin protein 1 (PfHP1) and histone deacetylase 2 (PfHda2) as key regulators of both processes, which provides a mechanistic link between parasite virulence and transmission.

Antigenic variation in P. falciparum is primarily mediated by switching the expression of the var genes, which encode the $P$. falciparum erythrocyte membrane protein 1 (PfEMP1). Each parasite expresses only one var gene at a time but can switch which gene is expressed to evade immunity. As histone modifications are known to contribute to changes in the expression of var genes, Brancucci, Bertschi et al. and Coleman, Skillman et al. investigated how two chromatin-associated proteins - PfHP1 and PfHda2, respectively - contribute to the regulation of antigenic variation. Brancucci, Bertschi et al. generated a conditional loss-of-function variant of PfHP1, which binds to the trimethylated lysine 9 residue of histone 3 (H3K9me3) and represses gene expression, whereas Coleman, Skillman et al. conditionally depleted $\mathrm{PfHda}$, which is a predicted histone deacetylase. Notably, the depletion of both proteins resulted in the derepression of many genes, and the strongest derepression corresponded to the var genes. The increased expression of multiple var genes was associated with decreased levels of $\mathrm{H} 3 \mathrm{~K} 9 \mathrm{me} 3$ in both mutants, and PfHP1-depleted parasites were shown to co-express several PfEMP1 variants. Together, these data show that gene repression that is mediated by $\mathrm{PfHP} 1$ and $\mathrm{PfHda} 2$ regulates antigenic variation.

In addition to the derepression of var genes, depletion of PfHP1 and PfHda2 also resulted in increased expression of several genes that are associated with sexual differentiation, particularly $p f a p 2-g$, which encodes the master regulator of gametocytogenesis - the transcription factor AP2-G. Using an in vitro gametocyte conversion assay, PfHda2-depleted parasites were found to produce more gametocytes than wild-type P. falciparum. Similarly, immunofluorescence assays showed that a higher proportion of PfHP1-depleted parasites differentiated into gametocytes than wild-type P. falciparum. These data show that PfHP1 and PfHda2 control sexual differentiation in $P$. falciparum by regulating the expression of $p f a p 2-g$.

Collectively, these results demonstrate that PfHP1 and PfHda2 regulate both antigenic variation and gametocytogenesis. This is consistent with a model in which PfHda2-mediated H3 deacetylation increases $\mathrm{H} 3 \mathrm{~K} 9 \mathrm{me} 3$ levels and

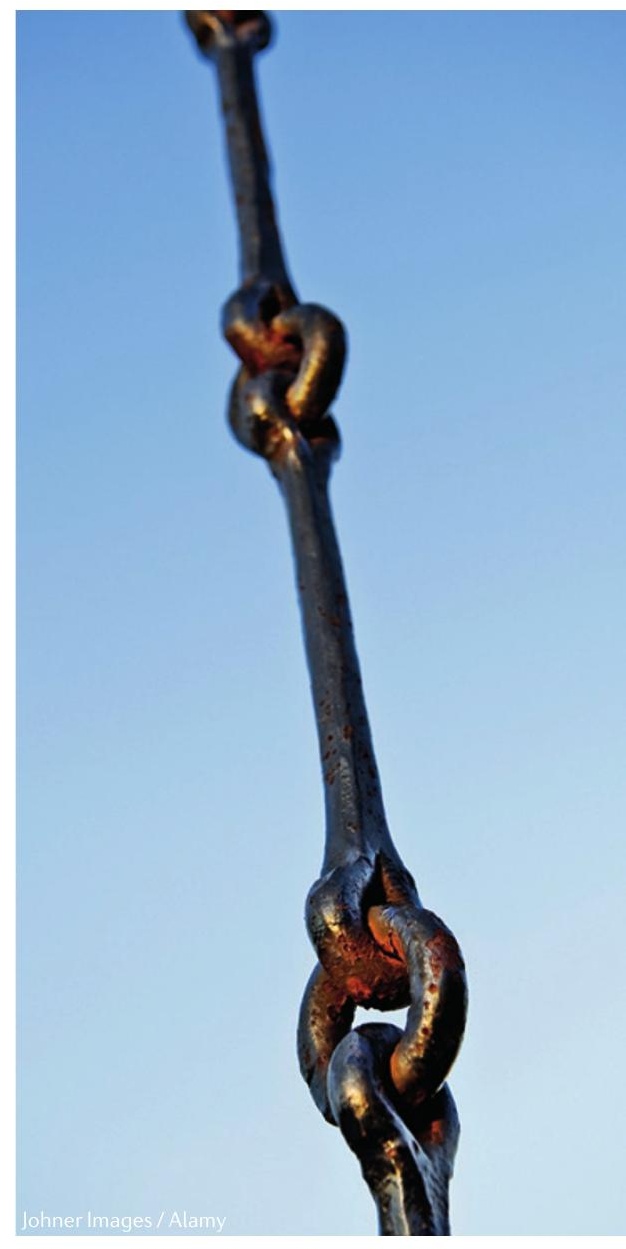

thereby functions as an upstream regulator that promotes the binding of PfHP1 to chromatin. Given the central role that these two proteins have in regulating the virulence and transmission of $P$. falciparum, it will be interesting to see whether PfHP1 and $\mathrm{PfHda} 2$ interact to form a silencing complex and whether such a complex constitutes a promising drug target to prevent the transmission of malaria.

Cláudio Nunes-Alves

ORIGINAL RESEARCH PAPERS Brancucci, N. M. B., Bertschi, N. L. et al. Heterochromatin protein 1 secures survival and transmission of malaria parasites. Cell Host Microbe 16, 165-176 (2014) | Coleman, B. I., Skillman, K. M. et al. A Plasmodium falciparum histone deacetylase regulates antigenic variation and gametocyte conversion. Cell Host Microbe 16, 177-186 (2014) 